\title{
Probing nonlinear rheology with inertio-elastic oscillations
}

\section{Citation}

Yao, Norman Y., Ryan J. Larsen, and David A. Weitz. 2008. "Probing Nonlinear Rheology with Inertio-Elastic Oscillations." Journal of Rheology52 (4): 1013-25. https:// doi.org/10.1122/1.2933171.

\section{Permanent link}

http://nrs.harvard.edu/urn-3:HUL.InstRepos:41511312

\section{Terms of Use}

This article was downloaded from Harvard University's DASH repository, and is made available under the terms and conditions applicable to Other Posted Material, as set forth at http:// nrs.harvard.edu/urn-3:HUL.InstRepos:dash.current.terms-of-use\#LAA

\section{Share Your Story}

The Harvard community has made this article openly available.

Please share how this access benefits you. Submit a story.

Accessibility 


\title{
Probing nonlinear rheology with inertio-elastic oscillations
}

\author{
Norman Y. Yao ${ }^{\text {a) }}$ \\ Department of Physics, Harvard University, Cambridge, Massachusetts 02138
}

Ryan J. Larsen

School of Engineering and Applied Sciences, Harvard University, Cambridge, Massachusetts 02138

David A. Weitz

Department of Physics and School of Engineering and Applied Sciences, Harvard University, Cambridge, Massachusetts 02138

(Received 20 October 2007; final revision received 25 April 2008)

\begin{abstract}
Synopsis
Many common materials display significant nonlinear rheological properties. Characterizing these properties can be done with a variety of methods. One such method uses inertio-elastic oscillations, which occur naturally in rotational rheometry as a consequence of a material's elasticity and the inertia of the rheometer. These oscillations have primarily been used to characterize linear viscoelastic properties. In addition to allowing for the imposition of stress-biased oscillations on short time scales, we demonstrate that extending this technique to nonlinear deformations provides accurate measurements of nonlinear material properties. Our experiments are performed on fibrin networks, which are well characterized and have dramatic nonlinear properties that are biologically significant. We compare the tangent moduli measurements of inertio-elastic oscillations with three standard methods of nonlinear rheology: forced oscillations about a prestress, a geometric interpretation of large amplitude oscillatory shears, and an extension of the linear viscoelastic moduli to the nonlinear regime. Inertio-elastic oscillations provide an accurate characterization of fibrin's nonlinear properties, and further, our measurements suggest that inertio-elastic oscillations provide the most straightforward method of distinguishing between nonlinear elasticity and dissipation at any given stress. In fact, we find that inertio-elastic oscillations provide the most accurate measurement of the subdominant loss component of our networks. (C) 2008 The Society of Rheology. [DOI: 10.1122/1.2933171]
\end{abstract}

\section{INTRODUCTION}

Rheological measurements are exceptionally important in characterizing a material's properties. Measurements are typically performed in the linear regime, where an oscillatory input will cause a single frequency material response whose amplitude is propor-

\footnotetext{
a) Author to whom correspondence should be addressed; electronic mail: nyao@fas.harvard.edu
} 
tional to that of the input. However, many materials also exhibit a nonlinear response that is both relevant and important. This is especially true for many biopolymer networks which can exhibit dramatic nonlinear rheological behavior [Storm and Pastore et al. (2005)]. The biological importance of this nonlinearity has inspired many in vitro studies of a variety of biological gels ranging from actin to collagen. Many of these in vitro gels show pronounced strain-stiffening behavior and accurately characterizing both energy storage and dissipation in this nonlinear stiffening regime remains an important experimental challenge.

There currently exist several methods to characterize a material's nonlinear properties. The most straightforward method is to extend the framework of the linear viscoelastic moduli $\left(G^{\prime}, G^{\prime \prime}\right)$ into the nonlinear regime. This method interprets large amplitude oscillatory shear data (LAOS) as though they were taken in the linear regime and fits the material's response to a single trigonometric function. Although the validity of $G^{\prime}, G^{\prime \prime}$ in the nonlinear regime is questionable, this method provides a simple way to capture the onset of nonlinearity. In the nonlinear regime, an explicit sinusoidal driving force results in a nonsinusoidal response that is not accurately governed by a single trigonometric function. This means that the linear viscoelastic moduli are no longer adequate for interpretations of LAOS data. When calculating $G^{\prime}, G^{\prime \prime}$, there exist a variety of methods to fit the material's response to a single trigonometric function, ranging from a Fourier transform to simple regressions; this adds to the confusion of interpreting $G^{\prime}, G^{\prime \prime}$ in the nonlinear regime. Large amplitude oscillatory shear data have also been interpreted in other ways to quantify material nonlinearity.

A popular method for analyzing LAOS data is the Fourier-transform method, which utilizes the relative intensities of higher harmonics as a measure of nonlinearity [Wilhelm and Maring et al. (1998); Kallus and Willenbacher et al. (2001)]. This method provides a precise assessment of the onset of nonlinearity. By calculating the relative amplitudes of the Fourier components, the deviation from linearity can be quantified with great accuracy. However, effectively incorporating higher harmonic information into calculations of nonlinear moduli remains a challenge, because during a single LAOS oscillation, the material is deformed over the entire range of stresses being studied. This range includes both linear and nonlinear deformations. Deconvoluting this information to determine the energy storage and dissipation at each stress or strain is a significant technical challenge. One recent approach to solving this problem has focused on a geometrical interpretation, whereby the nonlinear stress is broken into elastic and viscous components, leading to a generalized storage and loss modulus [Cho and Ahn et al. (2005)]. This method utilizes Lissajous plots of elastic stress vs. strain and viscous stress vs. strain rate to determine the nonlinear elasticity and dissipation of the material. Although this geometric method is useful, it does not completely overcome the low-signal limitation inherent in LAOS oscillations; for any oscillation, nonlinear deformations only occur during a limited portion of the cycle. A material's response can be dominated by the linear regime, making the ratio of nonlinear to linear signal quite small and thus requiring extremely precise measurements to extract the nonlinear behavior.

Perturbative methods can overcome both the low signal limitation and the deconvolution problem that exist in LAOS methods. Whereas a LAOS measurement deforms a material through many stress states, perturbative measurements seek to directly measure both energy storage and dissipation at a single stress. One such perturbative method, the differential measurement, uses forced oscillations about a steady prestress $\sigma_{0}$, which is utilized to bring the material to a specified deformation. The material properties of this state are then probed by perturbing the material with small stress oscillations of amplitude $\delta \sigma_{0} \ll \sigma_{0}$. In this method, the entire response signal is determined by the properties 
of the specific state. Thus, probing a nonlinear state with this method can overcome the low signal limitation of LAOS measurements. For the differential measurement, the small stress perturbation is accomplished by commanding the motor to superimpose an oscillatory stress above the prestress, so that the total stress is of the form $\sigma(t)=\sigma_{0}$ $+\left|\delta \sigma_{0}\right| \sin (\omega t)$. The resulting strain from the small oscillatory stress then takes the form $\delta \gamma(t)=\left|\delta \gamma_{0}\right| \sin (\omega t+\phi)$. As long as $\left|\delta \sigma_{0}\right| \ll \sigma_{0}$, the measured strain is a pure sinusoid and thus directly proportional to the applied oscillatory stress [Gardel and Shin et al. (2004)]. Thus, given that $\delta \sigma_{0}$ is sufficiently small, the deconvolution problem does not exist for the differential measurement. This perturbative method, though common, is complicated by the presence of irreversible flows in the material, which can severely complicate the interpretation of superposition data, even causing the moduli to be negative [Vermant and Walker et al. (1998)]. Obtaining a pure sinusoidal response requires that the steady state viscosity be sufficiently large, such that the irreversible flow is negligible on the time scale of oscillations. Moreover, irreversible flows might be associated with irreversible damage to the material's microstructure during the measurement, thereby introducing time dependence into the measurements. The risk of irreversible flows may be greater for a perturbative test than for LAOS tests because the material must be subjected to a steady nonlinear prestress for the entire duration of the sampling.

Both the long-time permanent deformation problem and negative modulus limitation can be overcome when the stress perturbation about the nonlinear state is supplied by the rotational inertia of a stress-controlled rheometer instead of the rheometer's motor. Immediately after the application of a steady shear stress, a sufficiently elastic sample will naturally experience stress oscillations caused by coupling between the sample's elasticity and the rheometer inertia-which we take to represent the combined inertia of the bearing, spindle, and geometry. The frequency and damping of these oscillations can be used to measure the elasticity and damping of the sample [Baravian and Quemada (1998); Ewoldt and McKinley (2007)]. Since inertio-elastic measurements utilize transient stress oscillations, they can be performed in short times without subjecting the material to long-time stresses that will significantly change the microstructure. Moreover, inertio-elastic oscillations overcome issues of interpretation related to the differential measurement because they are capable of distinguishing between dissipation resulting from irreversible flow and dissipation that is coupled with elastic deformations. Although previous work has used inertio-elastic oscillations to measure nonlinear properties, this method has yet to be used extensively, nor has it been compared to other current methods for probing nonlinearity [Baravian and Quemada (1998)].

We perform nonlinear measurements on an in vitro biological gel and demonstrate the usefulness and accuracy of inertio-elastic oscillations by comparing it with other nonlinear rheological methods, including an extension of the linear viscoelastic framework $\left(G^{\prime}, G^{\prime \prime}\right)$, a geometric interpretation of LAOS, and the differential measurement. We find excellent agreement between elasticity measurements using inertio-elastic oscillations and the other methods of measuring nonlinear tangent elasticity. Measures of nonlinear dissipation, however, were less reproducible between methods. Accurately measuring the subdominant component of a material's response, in our case the dissipation, is difficult with any test involving an oscillatory measurement. For example, we find that the differential loss modulus is extremely noisy and is even negative at certain points. However, utilizing inertio-elastic oscillations provides a measurement of the subdominant nonlinear dissipation that is much less noisy than the differential loss modulus. 


\section{EXPERIMENTAL}

A major focus of recent rheology research has been the nonlinear response of biopolymer networks. We utilize the biologically relevant and well-characterized material fibrin, which acts as the integral component of blood clots. Fibrin networks are an excellent model system with which to study the physics of biopolymer networks and are also an important part of the homeostatic process. Both the linear and nonlinear rheology of fibrin have been studied extensively in the past [Nelb and Gerth et al. (1976); Janmey and Amis et al. (1983); Spero and Smith et al. (2007)], making it a good starting point to test the effectiveness of inertio-elastic oscillations as a nonlinear rheological method. Most importantly here, the elasticity of these networks is highly nonlinear and extremely reproducible. Upon the addition of the enzyme thrombin $(0.5 \mathrm{U} / \mathrm{ml})$, the glycoprotein fibrinogen $(1 \mathrm{mg} / \mathrm{ml})$ is polymerized to form in vitro fibrin networks [Weisel (2005); Liu and Jawerth et al. (2006)]. This solution is gently mixed and loaded within 10 seconds onto the homemade steel bottom plate of a stress-controlled rheometer (AR-G2, TA Instruments). We utilize a steel bottom plate because the fibrin networks do not bind to the chrome-plated copper bottom plate of the rheometer, resulting in slip during measurements. All tests were performed with a two degree 20-mm-diameter cone plate tool and the moment of inertia of the entire rotating system, including the bearing, spindle, and geometry, is: $I=1.98^{*} 10^{-5} \mathrm{Nm} \mathrm{s}^{2}$.

We characterize the sample's polymerization, which lasts approximately 1 hour, by continuously measuring the linear viscoelastic moduli at a single frequency. During polymerization, we find that the linear viscoelastic moduli increase rapidly before reaching a plateau value. Upon full polymerization of the network, $G^{\prime}, G^{\prime \prime}$ both remain steady; we then measure the frequency dependence of the elastic and loss moduli. To ensure a linear response we maintain a maximum applied stress below $0.01 \mathrm{~Pa}$ and find that the linear moduli are frequency independent such that for all tested frequencies $(0.001-4.0 \mathrm{~Hz})$, $G^{\prime} \sim 20 \mathrm{~Pa}$ and $G^{\prime \prime} \sim 2 \mathrm{~Pa}$. Nonlinearity in the fibrin network's response occurs above a critical strain $\gamma_{C} \sim 0.1$ which corresponds to an applied stress amplitude of approximately $2 \mathrm{~Pa}$.

\section{RESULTS}

If the steady shear stress were ramped up slowly enough, the process of deformation would be quasi-static. For a viscoelastic solid, when the steady stress reaches its final value, the strain will also reach its final equilibrium value. However, the sudden application of a shear stress causes the material's strain response to overshoot its final equilibrium value, only to be pulled back by the sample's elasticity. This overshooting is a consequence of the rheometer's inertia and occurs as the rotational energy of the bearing is transferred to strain energy. This type of inertio-elastic oscillation results in an underdamped sinusoid, eventually reaching the equilibrium strain value, as shown in Fig. 1. However, it is important to note that not all materials exhibit under-damped oscillations and certain materials that do exhibit these oscillations may still experience too much irreversible flow to utilize this method effectively; thus, the method of inertio-elastic oscillations applies most readily to a viscoelastic solid. To better understand the inertioelastic oscillations of these viscoelastic networks we model the networks as a set of purely elastic springs and purely viscous dampers. We use a linear Kelvin-Voigt (KV) model, as seen in Fig. 2, which is represented as a purely viscous damper and a purely elastic spring connected in parallel. The governing equations for the model are: $\gamma_{\text {Total }}$ $=\gamma_{s}=\gamma_{d}$ and $\sigma_{\text {Total }}=\sigma_{s}+\sigma_{d}$, where the $s$ and the $d$ subscripts denote the strain/stress of the spring and damper, respectively. Thus, each component of the system feels the same 


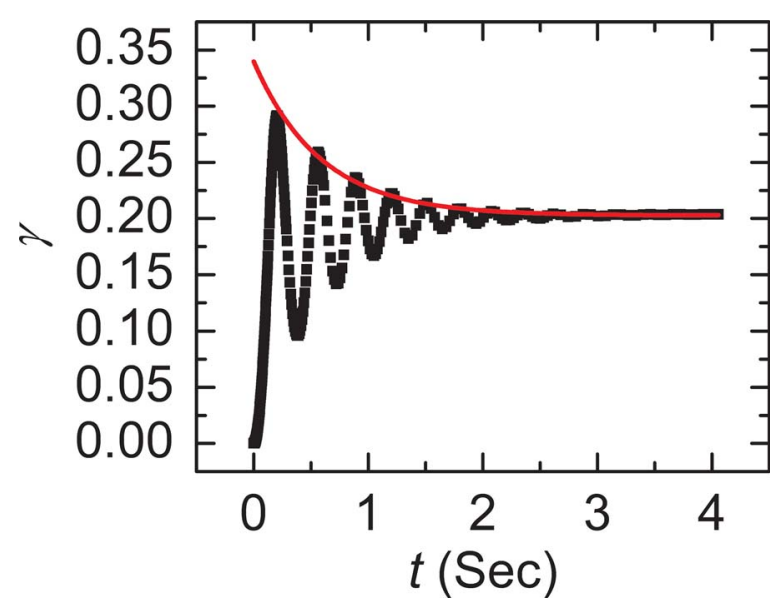

FIG. 1. Inertio-elastic oscillations in the nonlinear regime, obtained at a shear stress of $20 \mathrm{~Pa}$. Using regression, the damping is fit to an exponential decay (solid line).

strain while the total stress of the system is a sum of each of the stresses. Inertio-elastic oscillations occur in the KV model: considering the inertial contribution from the instrument in addition to the sudden application of a constant stress $\sigma_{C}$ results in a deformation approaching the deformation for the pure elastic material $\sigma_{C} / K_{C}^{\prime}$ [Baravian and Quemada (1998); Demiray (1994)]. Thus, the inertial contribution gives rise to a transient but does not affect the final steady state. To derive the elastic and loss modulus we begin by considering the equation of motion governing the bearing of the instrument: $I\left(F_{\sigma} / F_{\gamma}\right)\left(d^{2} \gamma / d t^{2}\right)=\sigma_{A}-\sigma_{S}$, where $I$ is the moment of inertia of the rheometer, $F_{\sigma}$ the proportionality factor between stress and torque such that $\sigma_{A}=F_{\sigma} \tau, F_{\gamma}$ the proportionality factor between strain and angular displacement such that $\gamma=F_{\gamma} \theta, \sigma_{A}$ the applied stress, and $\sigma_{S}$ the sample's resistant stress [Baravian and Quemada (1998)]. The solution to the $\mathrm{KV}$ model can be derived analytically by using the constitutive law, which is expressed as: $\sigma_{S}=E \gamma+\eta(d \gamma / d t)$. Coupling the equation of motion with the constitutive law yields an expression for critical elasticity. Only for an elasticity above the critical value of

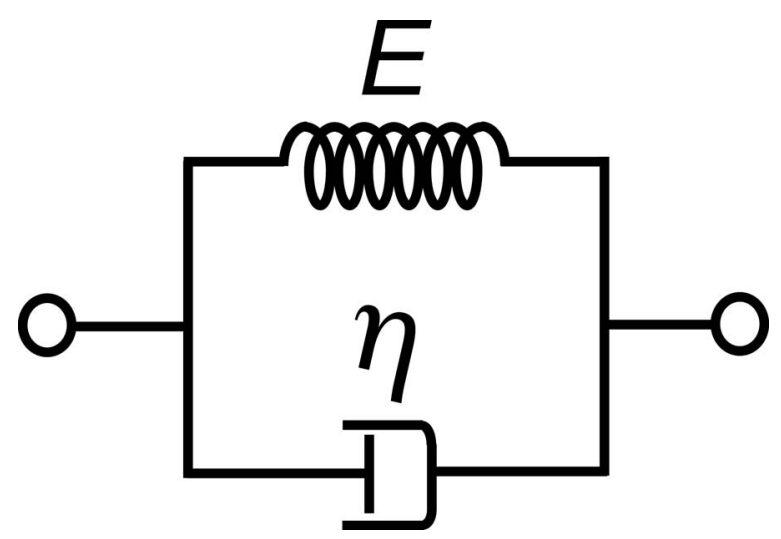

FIG. 2. The linear Kelvin Voigt model used in the derivation of the nonlinear moduli from inertio-elastic oscillations. 
$\eta^{2} / 4 a$ will oscillations occur, where $a=I\left(F_{\sigma} / F_{\gamma}\right)$. Solving the coupled differential equations under an applied step shear stress of $\sigma_{C}$ yields analytical solutions

$$
\sigma_{S}=\sigma_{C}\left[1-e^{-\beta t}\left(\cos (\omega t)-\frac{\beta}{\omega} \sin (\omega t)\right)\right]
$$

and

$$
\frac{d \gamma}{d \gamma}=\frac{\sigma_{C}}{a \omega} e^{-\beta t} \sin (\omega t)
$$

where $\beta=\eta / 2 a$ and $\omega=\sqrt{E / a-\beta^{2}}$. To find $\gamma$, we can simply integrate the second expression and impose the boundary condition that $\gamma(0)=0$ to find that

$$
\gamma=\frac{\sigma_{C}}{E}\left[1-e^{-\beta t}\left(\cos (\omega t)+\frac{\beta}{\omega} \sin (\omega t)\right)\right]
$$

[Zolzer and Eicke (1993); Baravian and Quemada (1998)]. To use the above results, we subtract the equilibrium strain and model the peaks using an exponential regression thereby finding the envelope of decay as shown in Fig. 1. We measure the oscillation frequency by averaging over the frequency of the first five cycles. Although the first few oscillation cycles may violate our assumptions of a linear KV model, we find that the variations in frequency are negligible in our case. In cases where the frequency variation is not negligible, we can avoid violations of the linear $\mathrm{KV}$ model assumption by utilizing later oscillations. The observed oscillations in $\sigma_{S}$ are less than ten percent of $\sigma_{C}$. This is an important condition because it allows us to think of the oscillations as small, linearizable, perturbations about a nonlinear state, thereby justifying our use of a linear KV model. Solving for the complex dynamic modulus from Eqs. (1)-(3) yields that $G^{*}=E$ $+i(\eta \omega)$ and thus the elastic modulus is given by $K_{c}^{\prime}\left(\sigma_{C}\right)=E=a\left(\omega^{2}+\beta^{2}\right)$ and the loss modulus by $K_{c}^{\prime \prime}\left(\sigma_{C}\right)=\eta \omega=2 a \omega \beta$. For inertio-elastic oscillations, the subdominant component is almost always the loss modulus because under-damped oscillations would be difficult to detect if the elasticity was subdominant. Although the simplest way to obtain these equations is with a KV model, similar equations for $K_{c}^{\prime}$, and $K_{c}^{\prime \prime}$ can also be obtained by calculating approximations from a general relaxation function [Struick (1967); Ferry (1980)]. As seen in Fig. 3, applying our equations yields nonlinear viscous and elastic differential moduli that increase with stress.

These calculations of inertio-elastic oscillations avoid the problems caused by the oscillatory measurements present in other nonlinear rheological methods. These problems include a single frequency response for $G^{\prime}, G^{\prime \prime}$, a noisy viscous-stress vs. strain-rate plot for the geometrical interpretation, and negative values of $K^{\prime \prime}$ in the nonlinear regime. In addition, another benefit of the inertio-elastic method is its ability to be applied when the required symmetry between strain and strain rate, which is required for the geometrical interpretation, cannot be guaranteed using a stress-controlled rheometer. However, since the method is based on the coupling of inertia to elasticity, it typically probes a single frequency, although additional frequencies can be accessed with variations in geometry factors and inertia [Baravian and Quemada (1998)].

We compare our results with other measurements of nonlinear viscoelasticity. The first is $G^{\prime}, G^{\prime \prime}$ measured in the nonlinear regime of fibrin by sweeping through stress amplitudes ranging from 0.1 to $20 \mathrm{~Pa}$ at a frequency of $0.1 \mathrm{~Hz}$. As shown in Fig. 4, both $G^{\prime}$ and $G^{\prime \prime}$ remain unchanged in the linear regime and exhibit dramatic increases in the nonlinear regime. Repeated tests show that the stress sweeps are reversible and the same moduli are obtained each time. Although the linear moduli are easily obtained with the 


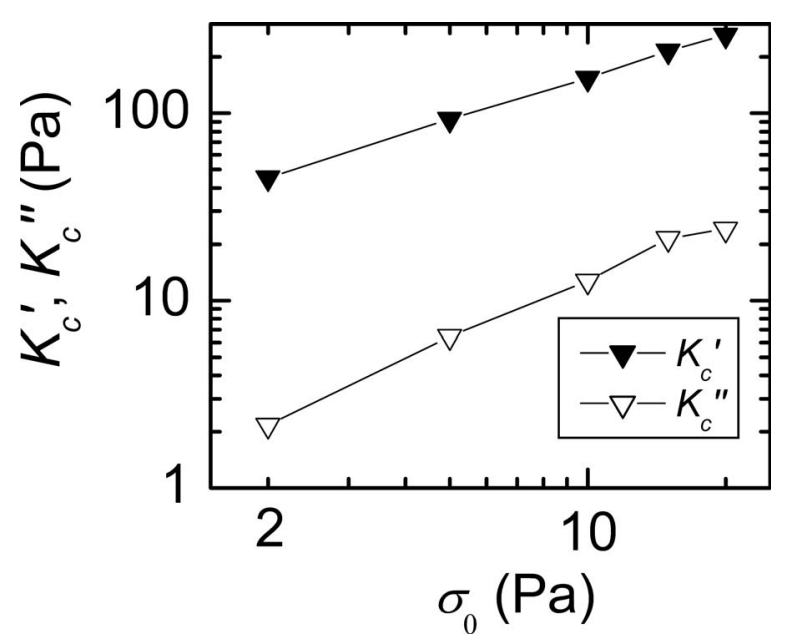

FIG. 3. Nonlinear differential moduli calculated from inertio-elastic oscillations.

rheometer software, and qualitatively capture the nonlinear stiffening of fibrin, they are not measurements of tangent moduli. Measurements of linear viscoelastic moduli assume certain conditions that are not met in the nonlinear regime. For example, measurements of $G^{\prime}, G^{\prime \prime}$ assume that an oscillatory input stress will result in a measured strain that is a pure sinusoid. This assumption is valid in the linear regime but is not valid in the nonlinear regime [Barnes et al. (1989); Larson (1999); Wilhelm (2002)].

The next method used to probe nonlinearity is the geometric interpretation of LAOS data. To apply the geometrical interpretation of LAOS, one requires perfect sinusoidal strain oscillations and therefore a rheometer with excellent strain control. This required symmetry between strain and strain rate will not necessarily exist for a stress-controlled rheometer in the nonlinear regime. The assumptions inherent to the linear viscoelastic moduli are avoided by plotting a stress-strain curve. The raw data is collected at a rate of 1000 points per second using a separate utility provided by TA Instruments. Lissajous

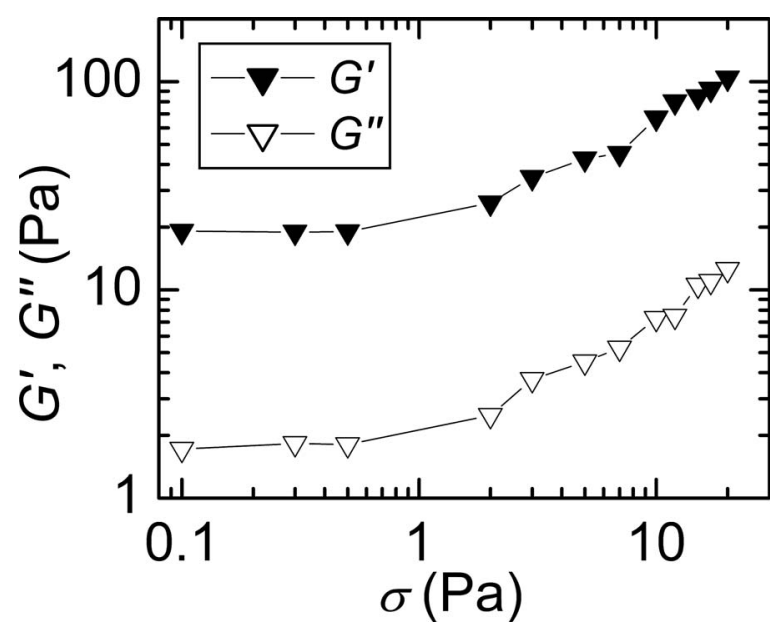

FIG. 4. The linear viscoelastic moduli, as calculated by the rheometer's onboard software, of $1 \mathrm{mg} / \mathrm{ml}$ fibrin obtained from a stress amplitude sweep at a frequency of $0.1 \mathrm{~Hz}$. 

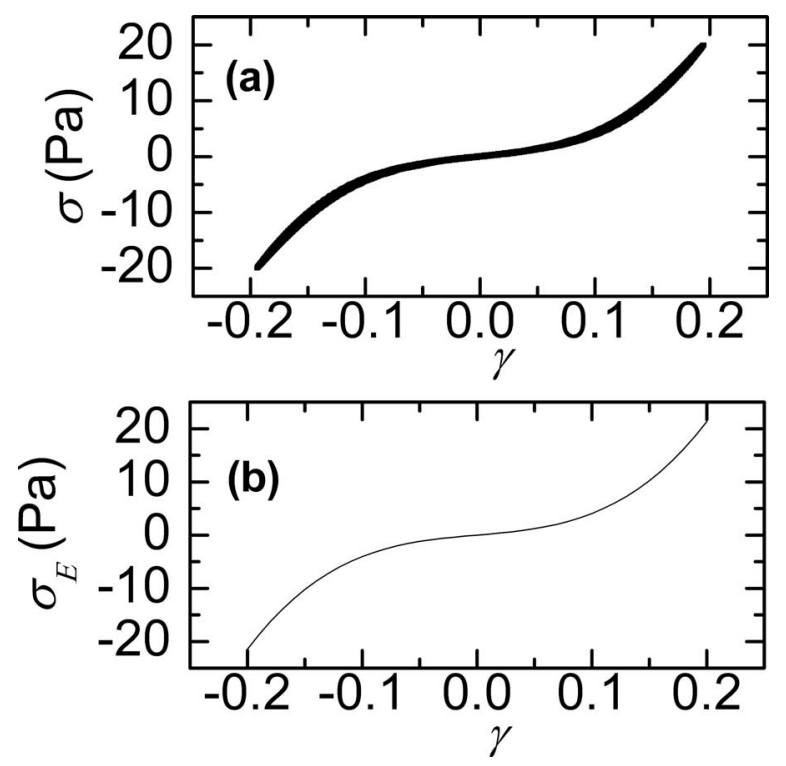

FIG. 5. Lissajous plot obtained from raw data of the instrument utility. (a) Strain-stiffening behavior of fibrin is shown by the nonellipsoidal shape. (b) Elastic stress vs. strain, from which $K_{s}^{\prime}\left(\sigma_{s}\right)$ is calculated.

figures were obtained by plotting $\sigma_{S}$ as a function of $\gamma$, where $\sigma_{S}$ was calculated by using the equation of motion for the bearing, $I\left(F_{\sigma} / F_{\gamma}\right)\left(d^{2} \gamma / d t^{2}\right)=\sigma_{A}-\sigma_{S}$. Values of $d^{2} \gamma / d t^{2}$ were calculated by taking forward derivatives of $\gamma$; before the second derivative, a MATLAB cubic spline smoothing algorithm was applied. At low frequencies inertial stresses were insignificant and $\sigma_{S}=\sigma_{A}$; however, at higher frequencies the inertia correction becomes important. Nevertheless, as expected, the high frequency Lissajous plots were identical with the low frequency Lissajous plot, over a range of $0.001-4 \mathrm{~Hz}$. This ability to extract moduli at a variety of stresses utilizing a single Lissajous figure is a benefit of the geometric interpretation. The shape of the Lissajous plot provides information about the mechanical properties of the material, with a linear response being an ellipsoid. Any deviation from a pure ellipsoid corresponds to a deviation from linearity. Further, the energy dissipated by the material can be obtained by integrating the area contained within the Lissajous figure. For the fibrin gel, the Lissajous curve encloses very little area because of the elastic nature of the network. Nonlinearity is evident in the Lissajous figure as the loop deviates dramatically from a pure ellipse as shown in Fig. 5(a). To find the differential elastic modulus at a given stress, we plot the elastic stress vs. strain curve in Fig. 5(b). The elastic stress at a specific strain is given by the average of the two stress values on the Lissajous plot [Cho and Ahn et al. (2005)]. Thus, using a single elastic stress-strain curve, the modulus as a function of stress amplitude $\left(\sigma_{s}\right)$ is $K_{s}^{\prime}\left(\sigma_{s}\right)$ $=[d \sigma / d \gamma]_{\sigma_{s}}$. To determine a loss modulus at each stress, we plot viscous stress as a function of strain rate, where the viscous stress is defined to be the absolute value of the difference between the elastic stress and the applied stress at a given strain [Cho and Ahn et al. (2005)]. However, because of the elastic nature of fibrin, the viscous component of the stress is extremely small causing the viscous stress vs. strain-rate Lissajous to be noisy and indeterminate. In addition, the measured strain is not a perfect sinusoid, which is also requisite for accurate strain-rate plots. The importance of accurately characterizing the subdominant component of a material's response at all stresses requires a method which quantifies both elasticity and dissipation. 


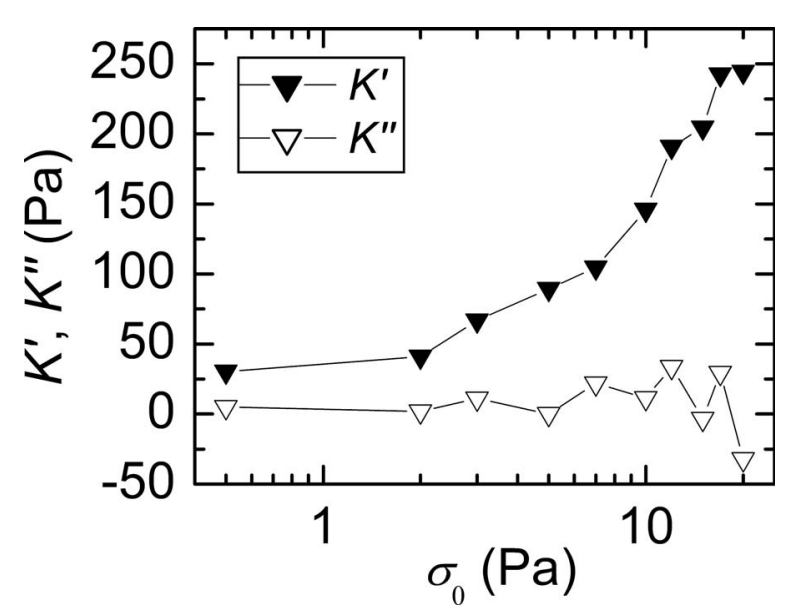

FIG. 6. The differential moduli as a function of prestress at $0.1 \mathrm{~Hz}$.

Finally, we examine forced oscillations about a prestress, which provide measurements of both viscoelastic moduli in the nonlinear regime. This differential measurement is similar to linear evaluations of $G^{\prime}$ and $G^{\prime \prime}$, but it is applied to a nonlinear state. Whereas $G^{\prime}$ and $G^{\prime \prime}$ are calculated using a ratio between stress and strain, the differential measurement probes the tangent moduli or the slope of the Lissajous curves. To calculate the differential elastic moduli we superimpose a small oscillatory stress $\delta \sigma_{0}(t)$ $=\left|\delta \sigma_{0}\right| \sin (\omega t)$ on a steady prestress $\sigma_{0}$. Thus the applied stress as a function of time is $\sigma(t)=\sigma_{0}+\left|\delta \sigma_{0}\right| \sin (\omega t)$. We measure the resultant oscillatory strain, $\delta \gamma(t)$ $=\left|\delta \gamma_{0}\right| \sin (\omega t+\phi)$. We confirm that the strain response is indeed linear and that the waveforms are pure sinusoids for all $\sigma_{0}$ provided that $\delta \sigma_{0}<\sigma_{0} / 10$. The differential elastic modulus is calculated by the rheometer and is given by $K^{\prime}\left(\sigma_{0}\right)=\left(\delta \sigma_{0} / \delta \gamma_{0}\right) \cos (\phi)$, while the differential viscous modulus is given by $K^{\prime \prime}\left(\sigma_{0}\right)=\left(\delta \sigma_{0} / \delta \gamma_{0}\right) \sin (\phi)$ [Gardel and Nakamura et al. (2006)]. The differential measurement provides a quantitative value for both moduli at each prestress. The elasticity exhibits similar trends when compared to the elasticity obtained from the two previous measures of tangent moduli. However, $K^{\prime \prime}$ is extremely noisy, even becoming negative at some prestresses, as shown in Fig. 6. It is expected that irreversible flows and permanent microstructure deformations will complicate differential measurements. While these fibrin samples demonstrate very little flow, the differential modulus is still extremely noisy since probing the loss modulus with an imposed oscillation would require an ability to accurately separate effects of relaxation from those of flow. This inability to separate the various effects and the time-dependent microstructural changes cause large variations in the reported differential loss modulus. These large variations could in part be caused by a difficulty of accurately measuring the phase angle. Although it is possible to improve the phase angle measurements by averaging over an increased number of cycles, we find that for our networks, the improvements are minor and the differential loss modulus is still negative at a variety of prestresses.

\section{DISCUSSION}

To verify the accuracy of $K_{c}^{\prime}$ and $K_{c}^{\prime \prime}$, we begin in the linear regime, and find that both viscous and elastic moduli from all four methods agree closely with one another. In the nonlinear regime, all of measurements of tangent elastic moduli, $K^{\prime}, K_{s}^{\prime}, K_{c}^{\prime}$ also agree 


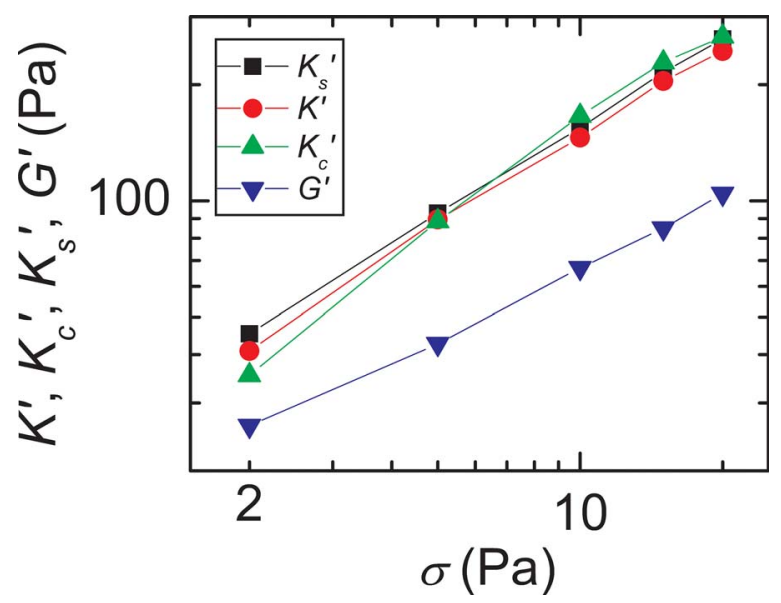

FIG. 7. A comparison of the storage moduli obtained from different methods, plotted as a function of maximum oscillatory stress amplitude for $G^{\prime}$, of $\sigma_{s}$ for $K_{s}^{\prime}$, of $\sigma_{0}$ for $K^{\prime}$, and of $\sigma_{C}$ for $K_{C}^{\prime}$.

closely with one another. By contrast, the extended linear elastic modulus does not agree with the other measurements because it is not a measure of the tangent modulus. $G^{\prime}$ is much lower than the other measures of elasticity as shown in Fig. 7; moreover, $G^{\prime}$ deviates further from the others as the nonlinearity increases. This is expected as increasing nonlinearity would result in additional departure from the linear viscoelastic assumption that the response strain is directly proportional to the input stress.

The internal consistency found within measurements of nonlinear elasticity does not extend to measurements of dissipation. In the nonlinear regime, $K^{\prime \prime}$ is dominated by noise, as shown in Fig. 8; moreover, although $G^{\prime \prime}$ and $K_{c}^{\prime \prime}$ show similar increasing trends, their magnitudes vary greatly. Of all methods used, only the inertio-elastic oscillations and the linear viscoelastic moduli provide low-noise, consistent values for both an elastic and loss modulus in the nonlinear regime. For both of these methods, the ratio of elasticity to dissipation remains constant such that at all stresses $G^{\prime} \sim 10 G^{\prime \prime}$ and $K_{c}^{\prime} \sim 10 K_{c}^{\prime \prime}$ as shown in Fig. 9. This factor is consistent with the ratio found in the linear regime of the fibrin

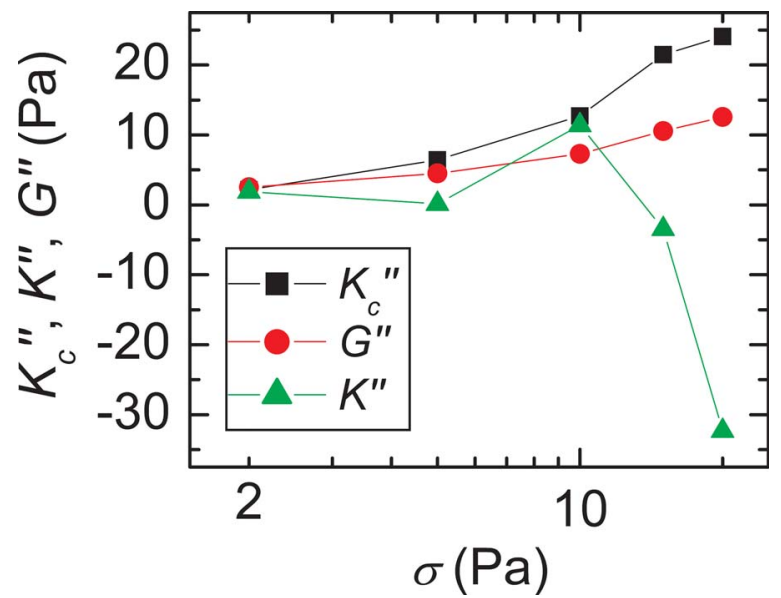

FIG. 8. A comparison of the various loss moduli obtained from different methods, plotted as a function of maximum oscillatory stress amplitude for $G^{\prime \prime}$, of $\sigma_{0}$ for $K^{\prime \prime}$, and of $\sigma_{C}$ for $K_{C}^{\prime \prime}$. The viscous stress vs. strain rate Lissajous are too noisy for $K_{s}^{\prime \prime}$ to be calculated. 


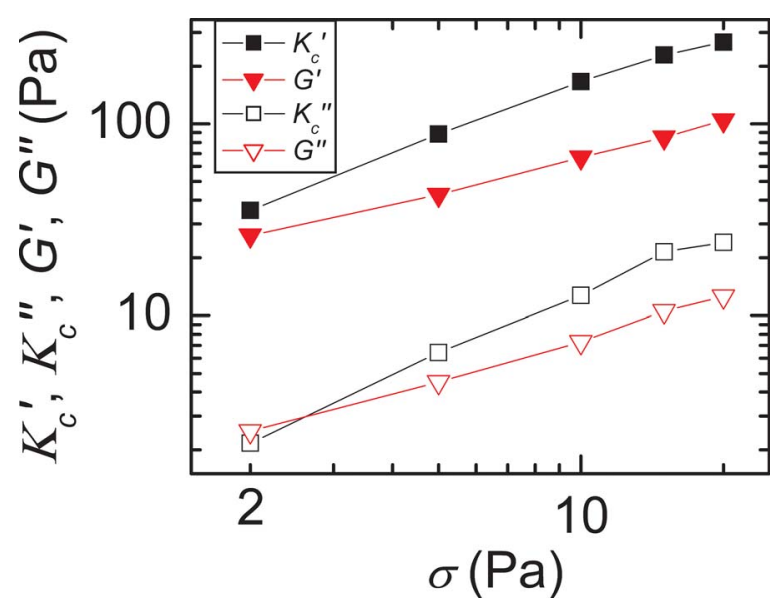

FIG. 9. The extended linear viscoelastic moduli and the differential moduli obtained from inertio-elastic oscillations, plotted against stress, depict similar trends.

gel where $G^{\prime} \sim 20 \mathrm{~Pa}$ and $G^{\prime \prime} \sim 2 \mathrm{~Pa}$. This suggests that both $G^{\prime \prime}$ and $K_{c}^{\prime \prime}$ correctly characterize the qualitative trends of the dissipation as a function of stress. However, quantitatively both linear viscoelastic moduli suffer from the assumption of a single frequency response, which is no longer valid in the nonlinear regime. Much of the information stored in the higher harmonics of the nonlinear response is lost for both $G^{\prime}$ and $G^{\prime \prime}$ since higher harmonic amplitudes are disregarded. We expect that the true nonlinear dissipation of the material is higher than the value suggested by $G^{\prime \prime}$ because our values of $K_{c}^{\prime \prime}$ are more than double those of $G^{\prime \prime}$ in the nonlinear regime. The consistency of the ratio $K_{c}^{\prime} / K_{c}^{\prime \prime}$ to $G^{\prime} / G^{\prime \prime}$ at all points in the nonlinear regime suggests that $K_{c}^{\prime \prime}$ is an accurate characterization of nonlinear dissipation. In fact, although the ratio $G^{\prime} / G^{\prime \prime}$ and $K^{\prime} / K^{\prime \prime}$ are approximately constant, the trend for both is to first decrease from $\sigma=2-15 \mathrm{~Pa}$ and then to increase slightly at $\sigma=20 \mathrm{~Pa}$, as seen in Fig. 10. This indicates that we can accurately characterize both a storage and loss modulus at each stress using inertio-elastic oscillations. Further, the oscillations associated with this method occur quickly, so that mea-

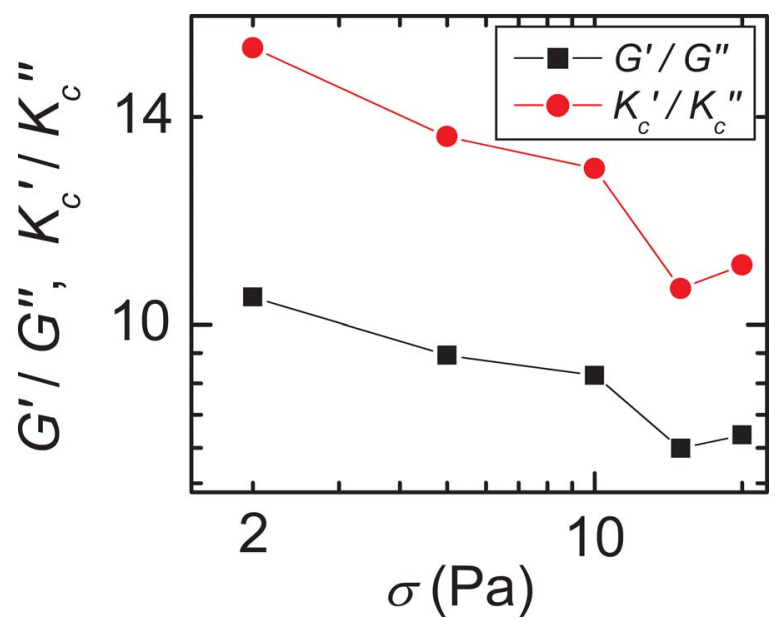

FIG. 10. The ratios $G^{\prime} / G^{\prime \prime}$ and $K_{C}^{\prime} / K_{C}^{\prime \prime}$, plotted against stress, show excellent qualitative agreement, first decreasing and then increasing slightly. 
surements can be made before significant irreversible microstructural changes occur to the sample as a result of nonlinear stress application. The simplicity of picking out the ringing frequency and the decay constant makes this method an elegant way to characterize the viscoelastic properties as a function of applied stress, in the nonlinear regime.

\section{CONCLUSION}

We find that measurements of nonlinear material properties with inertio-elastic measurements are consistent with standard methods of characterizing nonlinear elasticity. Inertio-elastic measurements were especially useful in measuring the subdominant response, in our case the dissipation, and did not suffer from the noise that dominated in both the geometric interpretation and forced oscillations about a prestress. While conventional methods for determining the elasticity and dissipation at each stress lack either consistency or accuracy, inertio-elastic oscillations provide both.

\section{ACKNOWLEDGMENTS}

The authors thank Gareth McKinley, Randy Ewoldt, and Karen Kasza for their useful discussions and insights. This work was supported by the NSF (DMR-0602684 and CTS-0505929), and the Harvard MRSEC (DMR-0213805).

\section{References}

Baravian, C., and D. Quemada, "Using instrumental inertia in controlled stress rheometry," Rheol. Acta 37(3), 223-233 (1998).

Barnes, H. A., J. F. Hutton, and K. Walters, An Introduction to Rheology (Elsevier, New York, 1989), pp. $107-132$.

Cho, K. S., and K. H. Ahn et al., "A geometrical interpretation of large amplitude oscillatory shear response," J. Rheol. 49(3), 747-758 (2005).

Demiray, H., "Viscoelastic model for arterial-wall materials," Int. J. Eng. Sci. 32(10), 1567-1578 (1994).

Ewoldt, R. H., and G. H. McKinley, "Creep ringing in rheometry or how to deal with oft-discarded data in step stress tests!” Rheol. Bull. 76, 4-6 (2007).

Ferry, J. D., The Viscoelastic Properties of Polymers (Wiley, New York, 1980), pp. 72-81.

Gardel, M. L., and F. Nakamura et al., "Stress-dependent elasticity of composite actin networks as a model for cell behavior," Phys. Rev. Lett. 96(8), 088102 (2006).

Gardel, M. L., and J. H. Shin et al., "Elastic behavior of cross-linked and bundled actin networks," Science 304(5675), 1301-1305 (2004).

Janmey, P. A., and E. J. Amis et al., "Rheology of fibrin clots. 6. Stress relaxation, creep, and differential dynamic modulus of fine clots in large shearing deformations," J. Rheol. 27(2), 135-153 (1983).

Kallus, S., and N. Willenbacher et al., "Characterization of polymer dispersions by Fourier transform rheology," Rheol. Acta 40(6), 552-559 (2001).

Larson, R. G., The Structure and Rheology of Complex Fluids (Oxford University Press, Oxford, 1999), pp. 217-229.

Liu, W., and L. M. Jawerth et al., "Fibrin fibers have extraordinary extensibility and elasticity," Science 313(5787), 634-636 (2006).

Nelb, G. W., and C. Gerth et al., "Rheology of fibrin clots. 3. Shear creep and creep recovery of fine ligated and coarse unligated clots," Biophys. Chem. 5(3), 377-387 (1976).

Spero, R. C., and B. Smith et al. "High throughput microbead rheology of fibrin gels," Biophys. J. 92(2), 521A-521A (2007).

Storm, C., and J. J. Pastore et al., "Nonlinear elasticity in biological gels," Nature (London) 435(7039), 
191-194 (2005).

Struick, L. C. E., "Free damped vibrations of linear viscoelastic materials," Rheol. Acta 6(2), 10-15 (1967).

Vermant, J., and L. Walker et al. "Orthogonal versus parallel superposition measurements," J. Non-Newtonian Fluid Mech. 79(2-3), 173-189 (1998).

Weisel, J. W., "Fibrous proteins: Coiled-coils, collagen and elastomers," Adv. Protein Chem. 70, 247-251 (2005).

Wilhelm, M., "Fourier-transform rheology," Macromol. Mater. Eng. 287(2), 83-105 (2002).

Wilhelm, M., and D. Maring et al., "Fourier-transform rheology," Rheol. Acta 37(4), 399-405 (1998).

Zolzer, U., and H. F. Eicke, "Free oscillatory shear measurements-An interesting application of constant stress rheometers in the creep mode," Rheol. Acta 32(1), 104-107 (1993). 